\title{
Cell Throughput Analysis of the Proportional Fair Scheduling Policy
}

\author{
Jin-Ghoo Choi and Saewoong Bahk \\ School of Electrical Engineering \& Computer Science \\ Seoul National University \\ Rm 314, Bldg 132, San 56-1, Shillim-dong, Kwanak-ku, Seoul 151-744, Korea \\ $\{$ cjk, sbahk\}@netlab.snu.ac.kr \\ TEL: $82-2-880-1813$ \\ FAX: 82-2-880-8214
}

\begin{abstract}
The fairness concept has been widely studied in the area of data networks. The most well-known fairness criterion, max-min fairness, gives priority to the minimum rate session. Kelly questioned its appropriateness in his works on the bandwidth sharing among the end-to-end flows and proposed another fairness criterion preferring short distance flows to enhance the overall throughput, which is called the proportional fairness (PF). A simple scheduler achieving this objective was introduced in wireless access networks and revealed that it can achieve a good compromise between cell throughput and user fairness. Though it has received much attention for some time, research on its performance mainly depended on computer simulations. In this paper, we analyze the PF scheduler to obtain the cell throughput which is a primary performance metric.
\end{abstract}

Keywords: Wireless networks, proportional fair scheduler, cell throughput

\section{Introduction}

Traditionally wireless access networks, especially cellular networks, have provided only voice service and were considered as an extension of wireline telecommunication systems. However, as demands for ubiquitous data services arose with the rapid growth of the Internet, wireless access networks and requirements placed upon them are evolving to support various high data rate services including multimedia. To accommodate these requirements, 3rd generation cellular systems such as wideband code division multiple access (W-CDMA) and cdma2000 have standardized enhanced radio access, and their core networks which currently consist of circuit switches and mobility supporting devices, are expected to be replaced by IP enabled components including routers [1].

In wireless data networks an important tool for resource management is the packet scheduler. Several works have shown that the scheduling policy can significantly affect system performances such as throughput, delay, fairness and 
loss rate in wireline and wireless domains [2]-[3]. Schedulers in wireless networks need to consider the unique characteristics of time-varying and location dependent channel conditions when contrasted with wired networks. Though many scheduling policies are proposed previously [4], they have the common weak point of assuming the channel to have only two states, "good" and "bad" states.

To know the channel state exactly, a BS requires fast feedback from mobile terminals about more detailed channel state information, which incurs a cost of increased signaling burden. For instance, the channel state can be divided into $2^{m}$ levels by using $m$ bits per feedback. This structure of wireless link state has been realized in the IS-856 standard for the downlink direction, which is known as the High Data Rate (HDR) system of Qualcomm [5].

Several schedulers for this architecture have been proposed. [6] maximizes cell throughput under the constraint that the normalized average throughput for each user is equal, where the normalization factor reflects users' required quality of service (QoS). The scheme proposed in [7] achieves the same objective with a different constraint where the allocated time portion of each user is assumed given. This scheduler can be shown to maximize the objective function (for example, based on utility) if it depends only on the signal-to-noise ratio (SNR). [6] and 7] use adaptive schemes with time-varying parameters, which can lead to convergence problems when implemented in real systems.

We consider schedulers exploiting the time-varying channel condition without explicit constraints. The maximum cell throughput can be obtained by serving the user with the best channel condition, however, it raises a serious fairness problem. The packet scheduler should meet a reasonable tradeoff between throughput and fairness. A scheduler achieving the PF criterion was introduced in [5], 8]. It has low complexity, which facilitates its implementation in real systems. Though it has received much attention for some time, the cell throughput obtainable by the PF scheduling policy has not been analyzed yet. Cell throughput is defined as the sum of each user's average throughput and is considered a primary performance metric for schedulers in wireless networks. In this paper, we analyze the PF scheduler and derive an approximate expression for cell throughput.

This paper is organized as follows. In Section II, we introduce the system and channel model. Section III analyzes the PF scheduler by adopting the linear and logarithmic models for relating the feasible rate and the SNR. Then we compare the simulation results with numerical analysis in Section IV, followed by concluding remarks in Section V.

\section{Model Description}

\subsection{System and Channel}

We consider the downlink channel of a single cell wireless access network where a BS serves $N$ mobile terminals (or users). The downlink structure is very similar to that of the IS-856 system. It is a single broadband channel shared by all 
users in the time division multiplexing manner. The BS exploits the pilot signal, which is pre-defined by the protocol, in the specified position of each time slot, and every mobile measures it to obtain the channel gain. The BS receives the fed back signal from all the users to collect the current channel status. Based on the channel information, the radio frequency scheduler selects a user among the active 1 ones to be served in the next slot. Though various schedulers are proposed in this context, among them we concentrate on the PF scheduler. A packet from the selected user is transmitted with the BS's full power, which is assumed fixed for all users.

When the transmission power of the BS is $P_{t}$, the receiving power of mobile user $i$ is given by $P_{i}=\left|h_{i}\right|^{2} P_{t}$, where $h_{i}$ is the channel gain written as

$$
h_{i}=\sqrt{c d_{i}^{-\alpha} s_{i}} z_{i}
$$

$c$ is a constant incorporating the transmission and receiving antenna gains, $d_{i}$ is the distance from the BS to user $i, \alpha$ is the path loss exponent (estimated to be about 4.0 in typical urban environments), $s_{i}$ is a random variable for the shadow fading effect, and $z_{i}$ represents the phasor sum of the multipath components. The shadow fading effect $s_{i}$ is known to follow the log-normal distribution with zeromean and the variance $\sigma_{s}^{2}(\mathrm{~dB})$ in the log-scale. The multipath fading effect $z_{i}$ is modeled as the second-order chi-square or exponential random variable with mean 1.0, which represents the Rayleigh fading channel.

Since we consider the single cell scenario, there is no inter-cell interference. Therefore, we can represent the SNR of user $i$ as $Z_{i}=P_{i} / P_{n}$ where $P_{n}$ is the background noise power which includes the thermal noise and other Gaussian interferences. 9 defined the median SNR at the cell edge, $l$, to represent the noise level of the wireless environment considered. For $l=c D^{-\alpha} P_{t} / P_{n}$ where $D$ is the radius of the cell, we obtain the average $2^{2}$ SNR of user $i$ as $E\left\{Z_{i}\right\}=\bar{Z}_{i}=l\left(\frac{D}{d_{i}}\right)^{\alpha} s_{i}$. Note that the received signal level follows the exponential distribution and the noise power is constant so that the SNR can also be modeled by the exponential random variable.

\subsection{PF Scheduler}

We briefly explain the operation of the PF scheduler. The average throughput of each user is tracked by an exponential moving average. At the beginning of each time slot, each user feeds back the channel state (or the feasible rate) to the $\mathrm{BS}$. The BS calculates the ratio of the feasible rate to the average throughput for each user, which is defined as the preference metric and is the key selection criterion. The user with the maximum preference metric will be selected for transmission at the next coming slot.

This is described formally as follows. In time slot $n$, the feasible rate of user $k$ is $R_{k}[n]$ and its moving average is denoted by $\tilde{R}_{k}[n]$. Then, user $k^{*}=$

\footnotetext{
${ }^{1}$ It means that its downlink queue is already backlogged.

${ }^{2}$ In this paper, we use both the notation $E\{\cdot\}$ and the overline to represent the average of a random variable.
} 
$\arg \max _{k} R_{k}[n] / \tilde{R}_{k}[n]$ is served in time slot $n$, and the average throughput of each user is updated by

$$
\tilde{R}_{k}[n+1]= \begin{cases}\left(1-\frac{1}{t_{c}}\right) \tilde{R}_{k}[n]+\frac{1}{t_{c}} R_{k}[n], & k=k^{*} \\ \left(1-\frac{1}{t_{c}}\right) \tilde{R}_{k}[n], & k \neq k^{*}\end{cases}
$$

where $t_{c}$ is the time constant for the moving average. It is clear that the PF scheduler affects relative preference to users with good channels as opposed to absolute preference.

In fast fading environments, the channel state of a user fluctuates around an average level in a random manner. When the number of users is small and all the users happen to be in bad state, then throughput in that slot will be low regardless of whoever is scheduled. On the other hand, when the number of users is large, some users will be in the good state with high probability. Therefore, we can obtain additional cell throughput gain by scheduling them first to utilize the characteristics of fast fading channels, which is called multi-user diversity.

\section{Analysis}

\subsection{Assumptions}

In this section we derive an approximate expression for the cell throughput under the PF scheduling policy. We make the following assumptions.

1. Users are distributed uniformly throughout the entire cell area.

2. Every session (or user) is always active in the downlink direction. We ignore the throughput loss due to the lack of data to be transmitted.

3. The distribution of the channel gain of user $i, h_{i}[n]$, does not depend on slot $n$ and it is constant for the slot duration.

4. Our model uses the ratio of the SNR to the average SNR as the preference metric instead of the original $\mathrm{PF}$ metric of the ratio of the feasible rate to the average rate (or throughput). That is, in time slot $n$, the user $k^{*}$ is served with $\arg \max _{k} Z_{k}[n] / \tilde{Z}_{k}[n]$ where $\tilde{Z}_{k}[n]$ denotes the exponential moving averaged SNR. Though this criterion is not exactly the same as that of the PF scheduler, they share the important characteristic of allocating almost the same portion of time slots to each user and allowing relative preference to users with good channels.

5. The feasible rate is a strictly monotonic increasing function of the SNR. It is also continuous while, in real systems, it takes values from a discrete set of supported rates. For example, the IS-856 system defines 11 feasible rates [5].

6. The average throughput and the average SNR are obtained by the time average rather than the moving average. For the throughput, $\tilde{R}_{k}[n+1]=$ $\frac{1}{n} \sum_{i=1}^{n} R_{k}[i] I_{k}[i]$, where the indicator function $I_{k}[i]$ is 1 if the user $k$ is scheduled in slot $i$ and 0 otherwise. The procedures to obtain the average SNR are the same. 
We add some remarks on assumptions 4 and 5. The feasible transmission rate depends on various factors such as the SNR, the degree of signal distortion during the transmission, the modulation scheme, the channel coding scheme, the hardware structure of the receiver, etc. Among these, the most important factor is the SNR, so we express the feasible rate as a function of the SNR aggregating other factors into a specific form.

We consider two popular models in this paper. They are the linear model and the logarithmic model. The linear model was widely adopted to describe CDMA systems though it is not accurate in very high SNR regions. In this model, our modified preference metric completely matches that of the PF scheduler. In the case of the logarithmic model, it does not coincide with the genuine preference metric of the PF scheduler and introduces errors in the analysis. This is evaluated by comparing the analysis with simulations.

\subsection{Cell Throughput of the PF Scheduler}

The redefined preference metric of the user $i$ is $\Gamma_{i}[n]=Z_{i}[n] / \tilde{Z}_{i}[n]$ in time slot $n$. When $n$ approaches infinity, we assume $\tilde{Z}_{i}[n]$ converges to a stationary value and denote it as $T_{i}$. Considering $Z_{i}[n]$ is independent of the slot time, we omit the time index $n$. Therefore, the preference metric is rewritten as

$$
\Gamma_{i}=\lim _{n \rightarrow \infty} \frac{Z_{i}[n]}{\tilde{Z}_{i}[n]}=\frac{Z_{i}}{T_{i}},
$$

which follows the exponential distribution.

We can write the long-term average throughput of user $i, \Phi_{i}$, which is the primary intermediate result to get the cell throughput, the product of the probability of scheduling user $i$ and its expected feasible rate when scheduled. We watch the "scheduled probability" of the user $i$ first. The scheduled user sees that its preference metric is larger than that of any other user. By denoting the maximum preference metric of all the users except $i$ as $\Gamma_{i-}$, we can write the scheduled probability as $\operatorname{Pr}\left\{\Gamma_{i}>\Gamma_{i-}\right\}$. Since $\Gamma_{i-}=\max \left\{\Gamma_{1}, \ldots, \Gamma_{i-1}, \Gamma_{i+1}, \ldots, \Gamma_{N}\right\}$ for $N$ users within the cell, its cumulative density function $(\mathrm{CDF}) F_{i-}(t)$ is given as

$$
F_{i-}(t)=\operatorname{Pr}\left\{\Gamma_{i-} \leq t\right\}=\prod_{j \neq i} F_{j}(t),
$$

where $F_{j}(t)$ is the $\mathrm{CDF}$ of $\Gamma_{j}$.

The feasible rate strongly depends on the SNR although the concrete relationship varies depending on the underlying technologies of the physical and link layers used. Since the SNR $Z_{i}$ is given as $T_{i} \cdot \Gamma_{i}$, the feasible rate $R_{i}$ is a function of $\Gamma_{i}$, which is denoted by $R_{i}=\xi\left(\Gamma_{i}\right)$. Since $\xi(\cdot)$ is a strictly monotonic increasing function of $\Gamma_{i}$, it always has the inverse function $\Gamma_{i}=\xi^{-1}\left(R_{i}\right)=\zeta\left(R_{i}\right)$. Then, the long-term average throughput of user $i$ is

$$
E\left\{\Phi_{i}\right\}=\operatorname{Pr}\left\{\Gamma_{i}>\Gamma_{i-}\right\} \cdot E\left\{R_{i} \mid \Gamma_{i}>\Gamma_{i-}\right\}
$$




$$
\begin{aligned}
& =\int_{0}^{\hat{R}} r \frac{d}{d r} \operatorname{Pr}\left\{R_{i} \leq r \text { and } \Gamma_{i}>\Gamma_{i-}\right\} d r \\
& =\int_{0}^{\hat{R}} r \frac{d}{d r} \operatorname{Pr}\left\{\Gamma_{i} \leq \zeta(r) \text { and } \Gamma_{i}>\Gamma_{i-}\right\} d r
\end{aligned}
$$

where $\hat{R}$ indicates the supported maximum feasible rate. After some manipulation, we obtain

$$
E\left\{\Phi_{i}\right\}=\int_{\zeta(0)}^{\zeta(\infty)} \xi(t) f_{\Gamma_{i}}(t) F_{i-}(t) d t
$$

where $f_{\Gamma_{i}}(t)$ denotes the probability density function (PDF) of $\Gamma_{i}$.

Consider the case where the SNR of user $i$ in time slot $n$ can be represented as the product of the constant $c_{i}$ and the random variable $C_{i}[n]$. That is, $Z_{i}[n]=$ $c_{i} C_{i}[n] . T_{i} / c_{i}$ is known to be the same for all $i$ 's when the following conditions are satisfied [10]: i) random variable $C_{i}[n]$ 's are i.i.d. regardless of users and time slots, ii) $C_{i}[n]$ 's are independent of the transmission power, and iii) the preference metric is a linear function of the SNR.

Since our model corresponds to this case with $c_{i}=\bar{Z}_{i}$ and $C_{i}[n]=\left|z_{i}\right|^{2}$, $T_{i} / c_{i}=T_{i} / \overline{Z_{i}}=\overline{\Gamma_{i}}$ is the same for all $i$ 's. Deleting the subscript $i$, we can see that, from (3), the PDF of $\Gamma_{i}$ is given as $f_{\Gamma_{i}}(t)=\frac{1}{\bar{\Gamma}} \exp \left(-\frac{t}{\bar{\Gamma}}\right)$ for $t \geq 0$ and, from (4), $F_{i-}(t)=\left(1-\exp \left(-\frac{t}{\bar{\Gamma}}\right)\right)^{N-1}$ for $t \geq 0$. Then, (5) can be rewritten as

$$
E\left\{\Phi_{i}\right\}=\int_{\zeta(0)}^{\zeta(\infty)} \xi(t) \frac{1}{\bar{\Gamma}} \exp \left(-\frac{t}{\bar{\Gamma}}\right)\left(1-\exp \left(-\frac{t}{\bar{\Gamma}}\right)\right)^{N-1} d t
$$

Next, we consider the function $R_{i}=\xi\left(\Gamma_{i}\right)$ in the linear and logarithmic forms.

Linear model. In this model the feasible rate is linearly proportional to the SNR. Past works adopted this model to describe CDMA systems and used it as an approximation to represent relations containing the small variation of SNR. When the relation is given as $R_{i}=\beta W Z_{i}$, where $\beta$ is a constant and $W$ is system bandwidth, we obtain the average throughput of user $i$ from (6) as follows.

$$
E\left\{\Phi_{i}\right\}=\frac{\beta W \overline{Z_{i}}}{N} \cdot N \int_{0}^{\infty} t e^{-t}\left(1-e^{-t}\right)^{N-1} d t,
$$

where $\frac{\beta W \overline{Z_{i}}}{N}=\frac{1}{N} E\left\{R_{i}\right\}$ represents the average throughput of user $i$ when the round robin scheduler is used. The second term is the auxiliary gain by using the $\mathrm{PF}$ scheduling and we define it as the multi-user diversity factor $M(N)$. After some manipulation, we have

$$
M(N)=N \sum_{m=0}^{N-1}\left(\begin{array}{c}
N-1 \\
m
\end{array}\right) \frac{(-1)^{m}}{(m+1)^{2}} .
$$

${ }^{3}$ Note that $\left|z_{i}\right|^{2}$ is the exponential random variable with the mean 1.0 regardless of $i$. 
The multi-user diversity factor increases rapidly with the increase in the number of users. This is owing to the very high SNR occasionally experienced during fast fading. Recognizing that the linear model overestimates the feasible rate in this region, we can see that the result has limited meaning in practical environments.

We derived a user's long-term average throughput assuming that the user's location is fixed or the shadowing effect is given. Now it needs to be averaged for all possible shadow fading and for the entire cell area and, then, multiplied by the number of users to obtain the cell throughput. If we denote the expectation of the shadowing by $E_{S}\{\cdot\}$,

$$
E_{S}\left\{\overline{\Phi_{i}}\right\}=\frac{\beta W}{N} M(N) E_{S}\left\{\overline{Z_{i}}\right\}=\frac{\beta W}{N} M(N) E_{S}\left\{s_{i}\right\} l\left(\frac{D}{d_{i}}\right)^{\alpha} .
$$

Representing the average over the entire area by $E_{A}\{\cdot\}$, we can express the cell throughput $\hat{T}_{\text {cell }}$ as

$$
\hat{T}_{\text {cell }}=N \cdot E_{A}\left\{E_{S}\left\{\bar{\Phi}_{i}\right\}\right\}=\beta W M(N) E_{S}\left\{s_{i}\right\} \cdot \Omega_{A}^{-1} \int_{A} l\left(\frac{D}{d_{i}}\right)^{\alpha} d A,
$$

where $A$ and $\Omega_{A}$ represent the cell and its area, respectively.

In this case, we can see that the cell throughput does not have a finite value. This means that the linear model is not accurate for very high SNR and produces unreasonably high data rates. Consequently, the extremely high throughput of users close to the BS gives rise to an infinite cell throughput. We can obtain the finite cell throughput by enforcing the distance between the BS and users to be larger than $D_{0}=\eta D(\eta<1)$. This reflects the fact that the transmission antenna of BS is, typically, apart from the ground. With this constraint, we can express the cell throughput as

$$
\hat{T}_{\text {cell }}=W \frac{4 l \beta}{2-\alpha} \frac{1-\eta^{2-\alpha}}{1-\eta^{2}} \exp \left(\left(\frac{\ln 10}{10 \sqrt{2}} \sigma_{s}\right)^{2}\right) M(N)
$$

by using $E_{S}\left[s_{i}\right]=2 \exp \left(\left(\frac{\ln 10}{10 \sqrt{2}} \sigma_{s}\right)^{2}\right)$. Henceforth, we use the normalized cell throughput $T_{\text {cell }}=\hat{T}_{\text {cell }} / W$ to make it independent of system bandwidth, which is the spectral efficiency of the cell in unit of bps/Hz.

Logarithmic model. Adaptive modulation schemes have been introduced to perform link adaptation. Among those, M-level quadrature amplitude modulation (M-QAM) is promising where $\mathrm{M}$ is typically set to the power of 2 . With the increase of $\mathrm{M}$, both the information bit rate and the bit error rate for the given SNR increase. So, the large M is used in good channel condition while the small $\mathrm{M}$ in bad condition. It was verified that, with M-QAM, the feasible transmission rate is related to the SNR in the logarithmic manner [9], [11]. In this paper, we adopt the form proposed in [9],

$$
R_{i}=W \log _{2}\left(1+\frac{Z_{i}}{K}\right)
$$


where $K$ is a constant depending on the system design and the target bit error rate required for reliable transmission. We call $K$ the system efficiency factor since the feasible rate for the same SNR varies according to $K$. When $K=1.0$, (12) becomes the well-known Shannon capacity formula.

Substituting $\xi\left(\Gamma_{i}\right)=\frac{W}{\ln 2} \ln \left(1+\frac{T_{i}}{K} \Gamma_{i}\right)$ into (6) $)$, we obtain the long-term average throughput of user $i$ as follows.

$$
\begin{aligned}
E\left\{\Phi_{i}\right\} & =\frac{W}{\ln 2} \int_{0}^{\infty} \ln \left(1+\frac{\overline{Z_{i}}}{K} t\right) e^{-t}\left(1-e^{-t}\right)^{N-1} d t \\
& =\frac{W}{\ln 2} \sum_{m=0}^{N-1}\left(\begin{array}{c}
N-1 \\
m
\end{array}\right)(-1)^{m} \int_{0}^{\infty} \ln \left(1+\frac{\overline{Z_{i}}}{K} t\right) e^{-(m+1) t} d t
\end{aligned}
$$

As $\int_{0}^{\infty} \ln (1+\mu t) e^{-\kappa t} d t=\frac{1}{\kappa} \exp \left(\frac{\kappa}{\mu}\right) \operatorname{expint}\left(\frac{\kappa}{\mu}\right)$ in [12], we can rewrite (13) as

$$
E\left\{\Phi_{i}\right\}=\frac{W}{\ln 2} \sum_{m=0}^{N-1}\left(\begin{array}{c}
N-1 \\
m
\end{array}\right) \frac{(-1)^{m}}{(m+1)} \exp \left(\frac{K}{\overline{Z_{i}}}(m+1)\right) \operatorname{expint}\left(\frac{K}{\overline{Z_{i}}}(m+1)\right)
$$

In [9], exp $(t) \operatorname{expint}(t)$ is closely approximated as $\nu_{1} \ln 2 \cdot \ln \left(1+\nu_{2} t^{-1}\right)$ for a wide range of $t$, where $\nu_{1}$ and $\nu_{2}$ are set to 1.4 and 0.82 , respectively.

Applying this approximation to (13), we obtain

$$
E\left\{\Phi_{i}\right\} \approx W \nu_{1} \sum_{m=0}^{N-1}\left(\begin{array}{c}
N-1 \\
m
\end{array}\right) \frac{(-1)^{m}}{(m+1)} \ln \left(1+\frac{\nu_{2}}{K(m+1)} \overline{Z_{i}}\right) .
$$

Taking the expectation of the shadow fading, we can approximate the above as

$$
E_{S}\left\{\overline{\Phi_{i}}\right\} \approx W \nu_{1} \sum_{m=0}^{N-1}\left(\begin{array}{c}
N-1 \\
m
\end{array}\right) \frac{(-1)^{m}}{(m+1)} \cdot E_{S}\left\{\ln \left(1+\frac{\nu_{2}}{K(m+1)} \overline{Z_{i}}\right)\right\} .
$$

Using $\overline{Z_{i}}=l\left(\frac{D}{d_{i}}\right)^{\alpha} s_{i}$, we need to calculate the form $E_{S}\{\ln (1+\mu s)\}$. We omit the subscript $i$ here for notational simplicity. In $[9], \operatorname{Pr}\{Y=\ln (1+\mu s) \leq y\}$ is shown to be close to $1-\frac{1}{2} \operatorname{erfc}\left(\frac{y-\ln \mu}{\frac{\ln 10}{10} \sqrt{2} \sigma_{s}}\right)$. This indicates that $E_{S}\{Y\}=E_{S}\{\ln (1+\mu s)\}$ is well approximated by the Gaussian random variable with the mean $\ln \mu$ and the standard deviation $\frac{\ln 10}{10} \sigma_{s}$. [9] also pointed out that it is valid only for $y \gg 1$. Since we are concerned with the mean rather than the CDF itself, we can use this approximation for our purpose.

However this gives an unsatisfactory result for small $\mu$. Especially, when $\mu$ is less than 1.0, we get a negative mean by the approximation while $Y$ is always larger than 0 . It motivates us to derive another approximation.

$$
E_{S}\{\ln (1+\mu s)\}=\ln (1+\mu)+\nu_{3},
$$

${ }^{4}$ expint $(t)$ is defined as $t \int_{1}^{\infty} \ln x \cdot e^{-t x} d x$ and called the exponential integral function. 


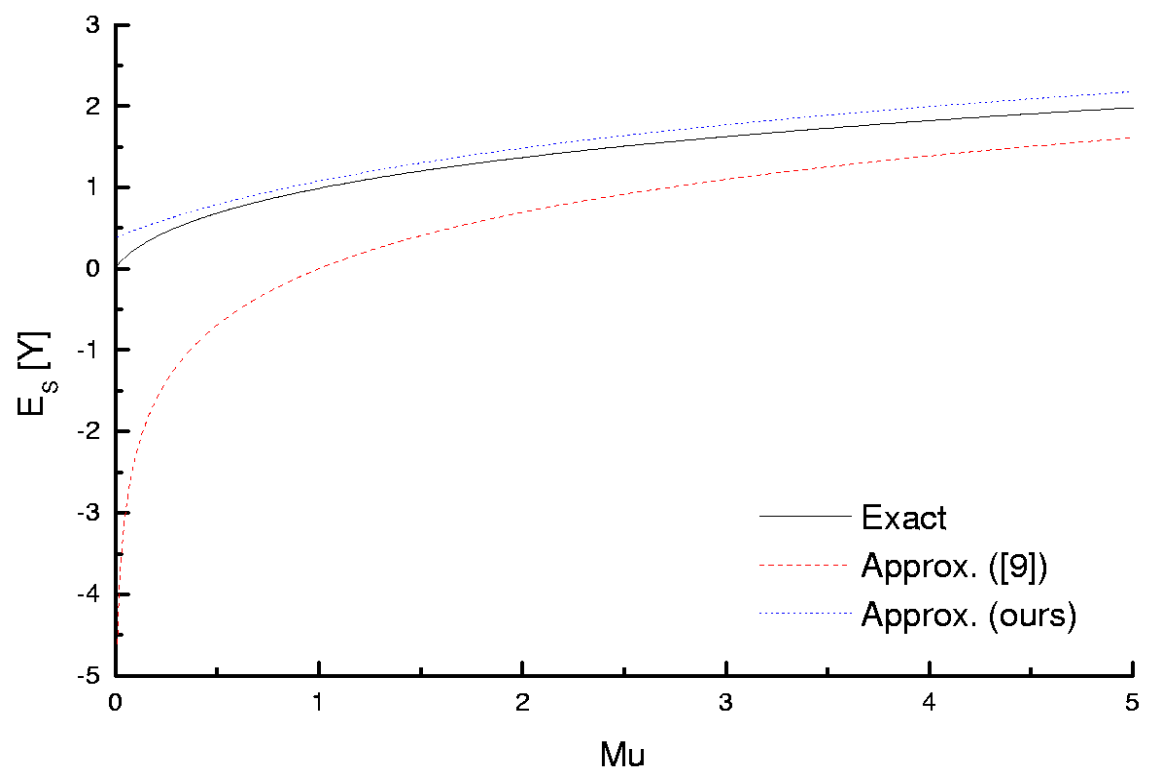

Fig. 1. Accuracy of the approximation for the average of the $\ln (1+\mu s)$.

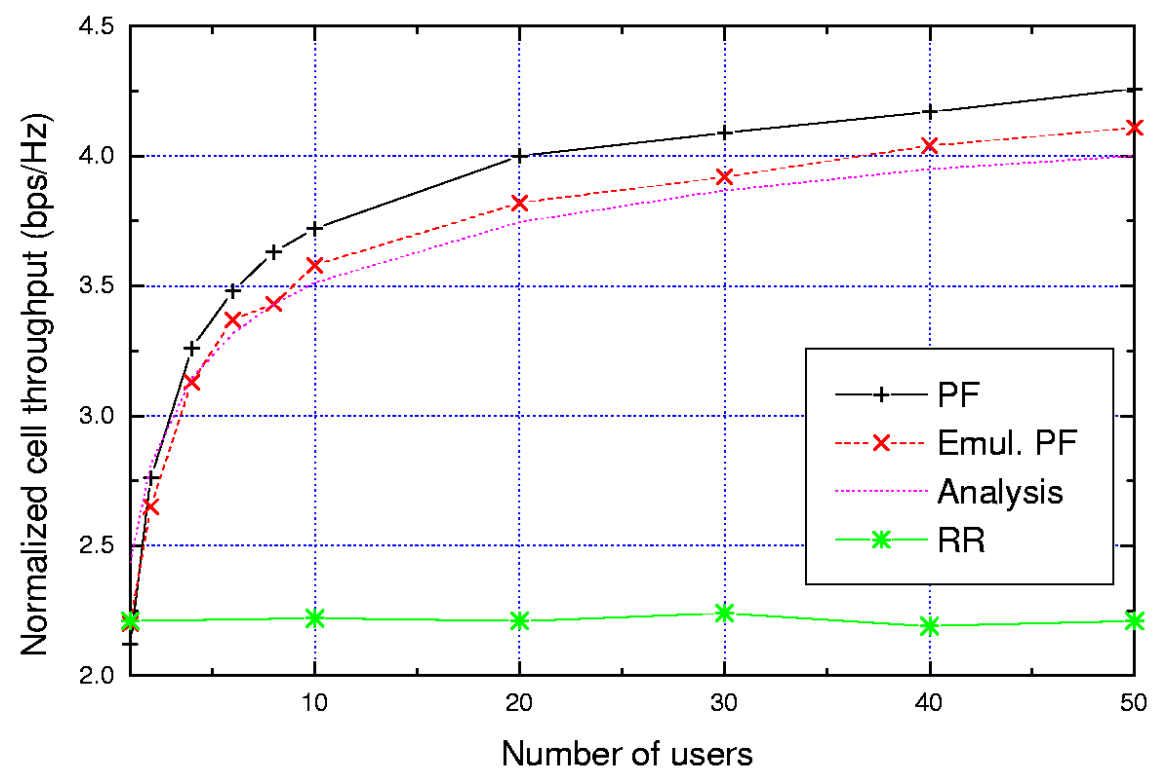

Fig. 2. Comparison of the analysis with simulations: $l=0(\mathrm{~dB})$ and $K=8(\mathrm{~dB})$. 
where $\nu_{3}=\frac{\ln 10}{10} \cdot \frac{\sigma_{s}}{\sqrt{2 \pi}}$. See the Appendix for the detailed derivation. Its accuracy is depicted in Fig. 11. Using this approximation, (15) becomes

$$
E_{S}\left\{\overline{\Phi_{i}}\right\} \approx W \nu_{1} \sum_{m=0}^{N-1}\left(\begin{array}{c}
N-1 \\
m
\end{array}\right) \frac{(-1)^{m}}{(m+1)} \cdot\left(\ln \left(1+a(m)\left(\frac{D}{d_{i}}\right)^{\alpha}\right)+\nu_{3}\right)
$$

where $a(m)=\frac{\nu_{2} l}{K(m+1)}$.

Finally, taking the average for the entire cell area, we can write the normalized cell throughput as

$$
T_{\text {cell }} \approx N \nu_{1} \sum_{m=0}^{N-1}\left(\begin{array}{c}
N-1 \\
m
\end{array}\right) \frac{(-1)^{m}}{(m+1)}\left(A(m)+\nu_{3}\right)
$$

where $A(m)$ is defined as

$$
A(m)=\Omega_{A}{ }^{-1} \int_{A} \ln \left(1+a(m)\left(\frac{D}{d_{i}}\right)^{\alpha}\right) d A=\frac{2}{D^{2}} \int_{0}^{D} r \ln \left(1+a(m)\left(\frac{D}{r}\right)^{\alpha}\right) d r
$$

We changed the dummy variable $d_{i}$ to $r$ for readability.

Now we integrate the form $\int x \ln \left(1+\mu x^{-\alpha}\right) d x$ to obtain $A(m)$ but this is difficult to do except in some special cases. $A(m)$ can be exactly calculated for the integer $\alpha \geq 3$. We present the result for $\alpha=4$, which is the most interesting case:

$$
A(m)=\ln (1+a(m))+2 a(m)^{\frac{1}{2}} \cdot \arctan a(m)^{-\frac{1}{2}} .
$$

Other values of $\alpha$ can be similarly handled by referring to [12].

\section{Simulation Results}

In this section, we present simulation results for the cell throughput under the PF scheduling policy and compare them with our analysis results. The throughput gap between the genuine PF scheduler and our emulated one is also manifested. Then we investigate the effect of various parameters on the throughput through numerical analysis.

A single cell scenario is considered in simulations and the cell radius is set to $1(\mathrm{~km})$. All mobile users are uniformly distributed over the entire cell area and the number of users varies from 1 to 50. The transmission power of BS is fixed at $10(\mathrm{~W})$. The path loss exponent $\alpha$ is 4.0 and the standard deviation of shadow fading $\sigma_{s}$ is $8.0(\mathrm{~dB})$. The median SNR at the cell edge, $l$, is set to $0(\mathrm{~dB})$ for hostile environments and $20(\mathrm{~dB})$ for good environments. The background noise power and the antenna gains are adjusted according to $l$.

The slot length for the downlink channel is 1.67 (msec). Each simulation runs for 1,000,000 slots and 1,000 outcomes of the same simulation runs are averaged to represent a single point of the graph. We only consider the logarithmic model for the relation between the feasible rate and the SNR since the linear model has only limited meaning in practical situations. 
Fig. 2 compares the analysis result with simulations where $l=0(\mathrm{~dB})$ and $K=8(\mathrm{~dB})$. The system efficiency factor $K=8(\mathrm{~dB})$ represents the feasible rate of the typical M-QAM system 5 We obtained very similar graphes for different $l$ 's and $K$ 's also, though they are not presented due to the lack of space. We observe that the simulation results for the PF scheduler are very close to those for the emulated PF scheduler6.

There is a tendency that the throughput of the PF scheduler is slightly higher than that of the emulated one. The analysis always tracks well the throughput of the emulated PF scheduler. Strictly speaking, our analysis slightly underestimates the throughput of the PF scheduler. The throughput of the RR scheduler is constant regardless of the number of users while that of the PF scheduler increases logarithmically with the increase of the number of users. Obviously the $\mathrm{RR}$ scheduler shows the same performance as the PF scheduler when $N=1$. The throughput gap between the two schedulers comes from the effect of the multi-user diversity for $N>1$.

\section{Conclusion}

In legacy wireless access networks, user fairness was not a pressing consideration. Several newly proposed systems, however, seriously consider user fairness and operate with packet schedulers that treat users differently according to the channel condition to achieve enhanced performance goals. The PF scheduler is one of the strong candidates targeted for balancing the cell throughput against user fairness by considering the channel condition.

In this paper we analyzed the PF scheduler to obtain an analytic expression for the cell throughput. We verified the accuracy of our analysis by comparing it with simulation results.

\section{References}

1. W. Mohr and W. Konhäuser: Access network evolution beyond third generation mobile communications. IEEE Commun. Mag. vol. 38, no. 12 (2000) 122-133

2. H. Zhang: Service disciplines for guaranteed performance service in packetswitching networks. Proc. IEEE vol. 83, no. 10 (1995) 1374-1396

3. A. K. Parekh and R. G. Gallager: A generalized processor sharing approach to flow control in integrated services networks: the single-node case. IEEE/ACM Trans. Networking vol. 1, no. 3 (1993) 344-357

4. Y. Cao and Victor O. K. Li: Scheduling algorithms in broad-band wireless networks. Proc. IEEE vol. 89, no. 1 (2001) 76-87

5. P. Bender, P. Black, M. Grob, R. Padovani, N. Sindhushyana, and S. Viterbi: CDMA/HDR: a bandwidth efficient high speed wireless data service for nomadic users. IEEE Commun. Mag. vol. 38, no. 7 (2000) 70-77

\footnotetext{
${ }^{5}$ On the other hand, when $K$ is $0(\mathrm{~dB})$ the feasible rate model follows the Shannon capacity formula and becomes an ideal system.

${ }^{6}$ Again the preference metric of the emulated PF scheduler is the ratio of the SNR to the average SNR, which was adopted for the facility of the analysis.
} 
6. S. Borst and P. Whiting: Dynamic rate control algorithms for HDR throughput optimization. in Proc. IEEE INFOCOM (2001) 976-985

7. X. Liu, E. K. P. Chong, and N. B. Shroff: Opportunistic transmission scheduling with resource-sharing constraints in wireless networks. IEEE J. Select. Areas Commun. vol. 19, no. 10 (2001) 2053-2064

8. A. Jalali, R. Padovani, and R. Pankaj: Data throughput of CDMA-HDR a high efficiency-high data rate personal communication wireless system. in Proc. IEEE VTC (2000) 1854-1858.

9. S. Catreux, P. F. Driessen, and L. J. Greenstein: Data throughputs using multipleinput multiple-output (MIMO) techniques in a noise-limited cellular environment. IEEE Trans. Wireless Commun. vol. 1, no. 2 (2002) 226-234

10. J. M. Holtzman: Asymptotic analysis of proportional fair algorithm. in Proc. IEEE PIMRC (2001) 33-37

11. A. J. Goldsmith and S.-G Chua: Variable-rate variable-power MQAM for fading channels. IEEE Trans. Commun. vol. 45, no. 10 (1997) 1218-1230

12. I. S. Gradshteyn and I. M. Ryzhik: Tables of Integrals; Series and Products. 4th ed. New York: Academic (1995)

\section{Appendix}

We show that $E_{S}\{\ln (1+\mu s)\} \approx \ln (1+\mu)+\nu_{3}, \nu_{3}=\frac{\ln 10}{10} \cdot \frac{\sigma_{s}}{\sqrt{2 \pi}}$, which is, in fact, the upper bound. Let $X$ be the log-normal random variable with the mean $0(\mathrm{~dB})$ and the variance $\sigma_{s}^{2}(\mathrm{~dB})$ in $\log$-scale. Since $Y=10 \log X$ follows the Gaussian distribution with the mean zero and the variance $\sigma_{s}^{2}$, from $f_{X}(x)=f_{Y}(y) \frac{d y}{d x}$, the PDF of $X$ is given as

$$
f_{X}(x)=\frac{c}{\sqrt{\pi}} \cdot \frac{1}{x} \exp \left(-(c \ln x)^{2}\right), \quad c=\frac{10}{\ln 10 \sqrt{2} \sigma_{s}} .
$$

Then,

$$
\begin{aligned}
E_{S}\{\ln (1+\mu s)\} & =\int_{0}^{\infty} \ln (1+\mu x) f_{X}(x) d x \\
& <\int_{0}^{1} \ln (1+\mu) f_{X}(x) d x+\int_{1}^{\infty} \ln ((1+\mu) x) f_{X}(x) d x \\
& =\ln (1+\mu) \int_{0}^{\infty} f_{X}(x) d x+\int_{1}^{\infty} \ln x \cdot f_{X}(x) d x \\
& =\ln (1+\mu)+\frac{\ln 10}{10} \cdot \frac{\sigma_{s}}{\sqrt{2 \pi}} .
\end{aligned}
$$

